\title{
Indigenous data sovereignty and COVID-19 data issues for American Indian and Alaska Native Tribes and populations
}

\author{
Aggie J. Yellow Horse ${ }^{1} \cdot$ Kimberly R. Huyser ${ }^{2}$
}

Accepted: 3 April 2021 / Published online: 9 April 2021

(C) The Author(s), under exclusive licence to Springer Nature B.V. 2021

\begin{abstract}
Indigenous Peoples in the United States have been experiencing disproportionate impacts of COVID-19. American Indian and Alaska Native persons are more likely to be infected, experience complications, and die from coronavirus. Evidence suggests that Indigenous persons have 3.5 times the incidence rate of non-Hispanic/ Latinx whites. Unfortunately, this is likely a gross underestimate because of a lack of reliable and accurate COVID-19 data for American Indian and Alaska Native populations. Multiple factors contribute to poor data quality including the lack of Indigenous representation in the data and rampant racial misclassification at both the individual and group levels. The current pandemic has shed light on multiple pre-existing issues related to Indigenous data sovereignty in data collection and management. We discuss the importance of centring Indigenous data sovereignty in the systemic efforts to increase COVID-19 data availability and quality. The federal and state governments must support and promote Tribes' rights to access data. Federal and state governments should also focus on bolstering their data availability and quality for aggregated data on AIAN populations and for providing disaggregated Tribal data to Tribes. Given the pivotal moment in the United States with ongoing and parallel pandemics of coronavirus and racism, we urge demographers and population scientists to reflect on the role of structural racism in data, data collection and analysis.
\end{abstract}

Keyword American Indian and Alaska Native populations · Indigenous Peoples · Indigenous data representation · Indigenous data sovereignty · COVID-19

Aggie J. Yellow Horse

ajnoah@asu.edu

Kimberly R. Huyser

kimberly.huyser@ubc.ca

1 School of Social Transformation, Arizona State University, Tempe, AZ, USA

2 Department of Sociology, The University of British Columbia, British Columbia, Canada 


\section{Introduction}

The coronavirus disease 2019 (COVID-19) has disproportionally affected racialised and minority populations in the United States, especially Black Americans and Latinx persons (Webb Hooper et al., 2020). However, relatively little is known about the disproportionate impact on Indigenous Peoples, due to the lack of available COVID-19 data for American Indian and Alaska Native (AIAN, hereafter) Tribes and populations (plural intended to honour multiple sovereign Native Nations). Indigenous Peoples are experiencing significant and disproportionate impacts of COVID-19. For example, the Navajo Nation has had the highest per capita coronavirus infection rate in the United States, and the top U.S. coronavirus hot spots are all on Tribal lands (Kristof, 2020). However, these appalling numbers and rates are likely still a gross underestimation due to data availability and quality issues.

COVID-19 data availability for AIAN Tribes and populations is limited through systemic erasure of Indigenous Peoples by inadequate Indigenous representation in data. At the early stage of the pandemic, only few states (AZ, CA, and MI) reported the number of confirmed COVID-19 cases and deaths for AIAN populations; but as of late June, 2020, about 26 states are now reporting race-specific numbers for AIAN populations (APM Research Lab, 2020). One might argue that the lack of available COVID-19 statistics for AIAN populations is driven by the relatively small population size of AIAN populations in each state. However, some states with larger AIAN populations have not yet provided race-specific COVID-19 statistics for AIAN populations. For example, $8.7 \%$ and $5.2 \%$ of the populations in South Dakota and North Dakota, respectively, are AIAN (US Census, 2020), but neither state has provided COVID-19 statistics for AIAN populations. Statistics from 23 states with sufficient data for AIAN populations have documented that the overall COVID-19 incidence among AIAN persons was 3.5 times higher compared to non-Hispanic white persons (Hatcher et al., 2020); thus, demonstrating the critical public health importance of data availability for AIAN Tribes and populations. However, it is important to note that impacts of COVID-19 on Tribes and AIAN populations substantially differ by state. Although AIAN populations make up only 9.6\% of New Mexico's total population (US Census, 2020), they account for about 50.8\% and $60.3 \%$ of the state's total COVID-19 confirmed cases and deaths (New Mexico Department of Health, 2020). Similarly, in Arizona, AIAN populations make up $4.5 \%$ of the state's population (US Census, 2020), but account for $8.0 \%$ and $19.6 \%$ of the confirmed COVID19 cases and deaths (Arizona Department of Health Services, 2020).

Even when AIAN populations are represented in COVID-19 data, it is of extremely poor quality and adds challenges to demographic approaches that are used to assess and mitigate the impacts of COVID-19 for Indigenous Peoples. Multiple factors contribute to poor data quality including individual and collective race misclassification issues. Misclassification occurs at the level of the individual when an individual is inaccurately categorised in reporting race and ethnicity. There are substantial race misclassification for AIAN persons where studies have found nearly $30 \%$ of AIAN patients had their race misclassified on their death certificate (Jim et al., 2014) and over $50 \%$ of AIAN patients had their race misclassified on hospital 
records (Bigback et al., 2015). Furthermore, misclassification occurs at the level of the individuals when the selection of multiple racial and ethnic categories are not permitted. For example, many states, including New Mexico, do not currently allow AIAN persons to simultaneously identify as both Latinx/Hispanic and AIAN. In Montana, American Indians are lumped into one category with Native Hawaiian and Pacific Islanders. While in other states, AIAN populations are lumped into other groups and classified as "others" in COVID-19 data. Collective misclassification occurs through the aggregation of Indigenous Peoples and other populations in to the "other" category. Through race misclassification of AIAN populations as "other," AIAN populations and Tribal Nations are rendered invisible in the public health discourse on the disproportionate impacts of COVID-19 on racialised and minority populations.

The current pandemic has shed light on multiple pre-existing issues related to Indigenous data sovereignty in data collection and management, and the federal and state governments must support and promote Tribes' rights to access data. It is reported that the Centers for Disease Control and Prevention have denied Tribal epidemiologists' requests for data but released the data freely available to the state agencies. And, a few states have even questioned Tribes' legal sovereignty (Tahir \& Cancryn, 2020). Such practices directly cause harm to Indigenous Peoples. It highlights the necessity of centring Indigenous data sovereignty in availability and quality of COVID-19 data and would provide the inherent authority of Tribal Nations to govern data about their peoples, lands, and resources (Kukutai \& Taylor, 2016). Consequently, Tribal authorities would be able to accurately impose data-informed mitigating policies for their citizens.

Since the start of the pandemic, states have employed different strategies for releasing data about AIAN populations and Tribal lands. For example, in Arizona, data release for Tribal lands or areas where AIAN populations makes up the majority have been suppressed (pending Tribal approvals). In Arizona, while some Tribes (e.g., Hopi Nation) decided to release their data at the zipcode level, many Tribes have not agreed to release their data to date (Curtis, 2020). On the other hand, New Mexico has been releasing zipcode-level data for Tribal lands without Tribal approvals. Such practices are extremely problematic above and beyond its violation of Indigenous data sovereignty of the Tribes in New Mexico. It can lead to breach of confidentiality of individual's information and identity of AIAN persons. It can also negatively impact Tribal economies and increase violence against their citizens. That is, given the relatively small population size of AIAN populations in specific places, we need to carefully consider the geographic scale for data release to protect the anonymity of individuals. Reliable and accurate COVID-19 statistics for AIAN populations at the aggregate level (i.e., state) are critical in ameliorating the disproportionate impact of COVID-19 on and eliminating disparities among Indigenous Peoples. The federal and state governments must respect and empower Tribes to control whether, how, and to what extent data about their peoples are released.

Federal and state governments should focus on bolstering their data availability and quality for aggregated data on AIAN populations and for providing disaggregated Tribal data to Tribes. There are substantial discrepancies in COVID-19 data. For example, in Arizona, three counties contain both non-tribal lands and tribal 
lands-i.e., Coconino, Navajo and Apache Counties. On May 5, 2020, the Arizona Department of Health Services reported 612 confirmed cases in Apache Countyaccounting for the whole county including all 18 zip codes that are inside and outside of the Navajo Nation boundary (Arizona Department of Health Services, 2020); but, on the same day, the Navajo Nation released that there were 656 confirmed cases from only 10 zip codes that fall on the Navajo Nation Tribal lands (The Navajo Nation, 2020). To improve quality of disaggregated data for Tribes, the data collection must include the efforts to include AIAN person's Tribal affiliation (Carroll et al., 2020). This is critical for Tribal Nations as many of their citizens may live outside of the boundaries of Tribal lands yet maintain intimate relations with families and relatives on Tribal lands (e.g., travels to the reservation). For example, about 53 percent of Navajo citizens reside outside of the Navajo Nation boundaries in both border towns and urban areas (i.e., 9 percent residing in border towns such as Gallup, NM and Winslow, AZ; and 44 percent residing away from the reservation and border towns) (Navajo Division of Health, 2013).

Given the pivotal moment in the United States with ongoing and parallel pandemics of coronavirus and racism, we urge demographers and population scientists to reflect on the role of structural racism in our own fields beyond the argument for high cost of data collection for "numerically-small" populations. That is, we must explicitly acknowledge and be held accountable for the ways that population science have played in the systemic erasure of Indigenous Peoples in data and enabled the historical marginalisation, dispossession of lands, and displacement of Indigenous Peoples. We also urge demographers and population scientists to actively engage in discussions on meanings and implications of justice in the Indigenous representation in data, and ways to dismantle barriers for Indigenous representation in data through systematic investment in increasing data collection with Indigenous Peoples and communities; fostering Indigenous demographers and population scientists through institutional supports for training; and abiding commitment for building meaningful partnerships with Tribal Nations and Indigenous communities in the United States and globally.

\section{References}

APM Research Lab. (2020). The color of coronavirus: COVID-19 deaths by race and ethnicity in the US. APM Research Lab. https://www.apmresearchlab.org/covid/deaths-by-race. Accessed 12 October 2020.

Arizona Department of Health Services. (2020). COVID-19 data dashboard by demographics. Arizona Department of Health Services.

Bigback, K. M., Hoopes, M., Dankovchik, J., Knaster, E., Warren-Mears, V., Joshi, S., \& Weiser, T. (2015). Using record linkage to improve race data quality for American Indians and Alaska natives in two pacific northwest state hospital discharge databases. Health Services Research, 50(Suppl 1), 1390-1402.

Carroll, S.R., D. Rodriguez-Lonebear, R. Akee, A. Lucchesi, and J.R. Richards. (2020). Indigenous Data in the Covid-19 Pandemic: Straddling Erasure, Terrorism, and Sovereignty. In items: Insights from the social sciences. Social Science Research Council. https://items.ssrc.org/covid-19-and-the-socialsciences/disaster-studies/indigenous-data-in-the-covid-19-pandemic-straddling-erasure-terrorismand-sovereignty/. Accessed 12 October 2020. 
Census, U. S. (2020). 2014-2018 American community survey 5-year estimates. US Census Bureau.

Curtis, C. (2020). Hopi tribe releases ZIP code data, but COVID-19 data for Arizona's 22 communities is confusing. Arizona Republic. https:/www.azcentral.com/story/news/local/arizona-health/2020/ 06/04/coronavirus-arizona-indigenous-communities-cases-covid-19-data-hopi-navajo/3091650001/. Accessed 12 October 2020.

Hatcher, S. M., Agnew-Brune, C., Anderson, M., Zambrano, L. D., Rose, C. E., Jim, M. A., Baugher, A., Liu, G. S., Patel, S. V., \& Evans, M. E. (2020). COVID-19 among American Indian and Alaska native persons-23 states, January 31-July 3, 2020. Morbidity and Mortality Weekly Report, 69(34), 1166-1169.

Jim, M. A., Arias, E., Seneca, D. S., Hoopes, M. J., Jim, C. C., Johnson, N. J., \& Wiggins, C. L. (2014). Racial misclassification of American Indians and Alaska natives by Indian health service contract health service delivery area. American Journal of Public Health, 104(Suppl 3), S295-S302.

Kristof, N. (2020). The top U.S. coronavirus hot spots are all Indian lands. New York Times. https://www. nytimes.com/2020/05/30/opinion/sunday/coronavirus-native-americans.html. Accessed 12 October 2020.

Kukutai, T., \& Taylor, J. (2016). Indigenous data sovereignty: Toward an agenda. ANU Press.

Navajo Division of Health. (2013). Navajo population profile, 2010 U.S. census. Navajo Division of Health.

New Mexico Department of Health. (2020). COVID-19 in New Mexico. New Mexico Department of Health.

Tahir, D., Cancryn, A. (2020) American Indian tribes thwarted in efforts to get coronavirus data. POLITICO. https://www.politico.com/news/2020/06/11/native-american-coronavirus-data-314527. Accessed 12 October 2020.

The Navajo Nation. (2020). May 10, 2020: 149 new cases of COVID-19 and two more deaths reported. Office of the President and Vice President of the Navajo Nation.

Webb Hooper, M., Nápoles, A. M., \& Pérez-Stable, E. J. (2020). COVID-19 and racial/ethnic disparities. Journal of the American Medical Association, 323(24), 2466-2467.

Publisher's Note Springer Nature remains neutral with regard to jurisdictional claims in published maps and institutional affiliations. 